\title{
Application of Industrial Control Configuration Software WinCC in the Monitoring System of Ship Steering Gear
}

\author{
Jiayu Chen ${ }^{a}$, Hongbo Wang ${ }^{b}$ and Hongjun Lv ${ }^{c}$ \\ School of JiLin University, Changchun 130000, China \\ achen_jiayu_happy@163.com, bwang_hongbo@jlu.edu.cn, clvhj15@mails.jlu.edu.cn
}

\begin{abstract}
In this paper, develop the monitoring system of ship steering gear with configuration software WinCC. It communicates with PLC controller through OPC protocol, and as a WinCC server of the distributed system, providing the process data to the WinCC clients. It takes Kawasaki pump controlled hydraulic steering gear as the monitoring object. It has a humanized human machine interface. The operators can look over the real-time running status of the ship steering gear, and find out the problems quickly and maintain timely according to the alarm information. It can also provide the historical records of important data for analysis.
\end{abstract}

Keywords: Steering gear monitoring system; OPC communication; WinCC distributed system; Human machine interface.

\section{Introduction}

Steering gear is a kind of large deck machinery, which is a very important equipment to keep and change the direction of the movement of the ship in the sea. The reliability, stability and maneuverability of steering gear monitoring system will directly affect the economy and safety of the ship in the course of navigation.

With the development of automatic monitoring technology, the requirement of ship equipment automation degree is increasingly high. Industrial control configuration software WinCC is an integrated human machine interface monitoring and management system, and WinCC's full name is window control center. It includes variable management, graphics editor, alarm records, variable records, global script and other functions, so that it has the high performance of the process of coupling, fast screen refresh and reliable data management. Industrial control configuration software WinCC is used for configuring human machine interaction center of the ship steering gear monitoring system. It displays various parameters of the units on the monitoring interfaces in order that the operators can understand the working condition of the rudder and send the instruction to control the steering gear.

\section{The communication with PLC controller through OPC protocol}

\subsection{OPC technology introduction.}

OPC's full name is OLE for Process Control, which acts as a bridge between the applications based on Windows and on-sited process control. Original OLE object is linking and embedding. With the popularity of OLE, now OLE includes many new features, such as the unified data transmission, structured storage and automation. It has become a specification, which is independent of the computer language, operating system and hardware platform. It is the further promotion of the concept of the object-oriented programming. OPC standard based on OLE technology. Its establishment is completed by providing a series of standard OLE/COM interface. It provides a standard data access mechanism for the industrial control field.

OPC adopts the client / server mode. It attributes the task of development and access of the interface to a hardware manufacturer or a third party manufacturer and provides to the users in the form of OPC server. Resolve the contradiction between the software and hardware manufacturers, achieve the system integration, and improve the openness and interoperability of the system. 


\subsection{OPC server and PLC configuration.}

Simatic Net, as a server of OPC communication. Firstly, configure the PC Station hardware frame with the Station Configuration Editor, add the "OPC Server" to the number one slot and the "IE General" to the number three slot, and set up the network parameters.

Secondly, configure the Configuration Console, switch the mode of Ethernet card from the "PG mode" to the "Configuration mode", and set "Index"with"three". In the Access Points setting item, we set the "S7ONLINE" with "PC internal (local)", it makes preparations for the PC Station configuration download.

Next, configure the PC Station in the STEP7, that is to say, insert a "PC Station", and add the same hardware configuration and configure the same network parameters as Configuration Editor Station in the PC Station. Then establish a connection in the NetPro network configuration center, compile without error, and the configuration can be downloaded to the PC machine.

After the download completely, you can open the Station Configuration Editor window to check the status of the component, if you can see the connection icon in the conn column of OPC Server slot, it shows that the connection is activated.

\subsection{Set up OPC connections in the WinCC project.}

WinCC, as a client of OPC communication, can read the data from OPC server when the OPC connective configuration is accomplished. Firstly, add new driver "OPC.chn" in the WinCC variable manager. Then, select the OPC server in the system parameter options of the driven and connected of OPC. Finally, add data variables of transmission, and can also create your own new variables. The setting of external variables of the WinCC is accomplished.

\section{Interface design of the ship steering monitoring system}

\subsection{Working condition introduction.}

In this paper, the Kawasaki pump controlled hydraulic steering gear is taken as the monitoring object. The main pump is running continuously according to the given direction at work, the direction and emissions of suction and exhaust are controlled by the size and direction of the displacement of the deviation from the center of the variable pole. And the direction and size are related to the rudder angle deviation signal which is obtained from comparing of the command rudder angle signal and the actual rudder angle signal.

Steering gear has three kinds of working conditions. Single pump and four cylinders working condition, it is suitable for open water in normal navigation; Double pumps and four cylinders working condition, it is appropriate for quick steering, as ships steam in and out of the port or narrow waterway; Single pump and double cylinders working condition, it is used when a cylinder leaks the oil.

\subsection{Interface design overview.}

Starting from the overall layout and manageability, and thinking about the different functions, the monitoring platform will be divided into four interfaces, "Running Status", "Instrument Value", "Alarm" and "Historical Data". According to clicking the corresponding function buttons to switch among the interfaces, the interface functions are accomplished by adding various types of controllers and writing a script.

\subsection{Monitoring interfaces explanation.}

(1) The "Running Status" interface. When the system is running, the "Running Status" interface is the default display, this interface real-time displays the steering mode and the working status of the hydraulic system of the two units. As shown in the Fig. 1. 


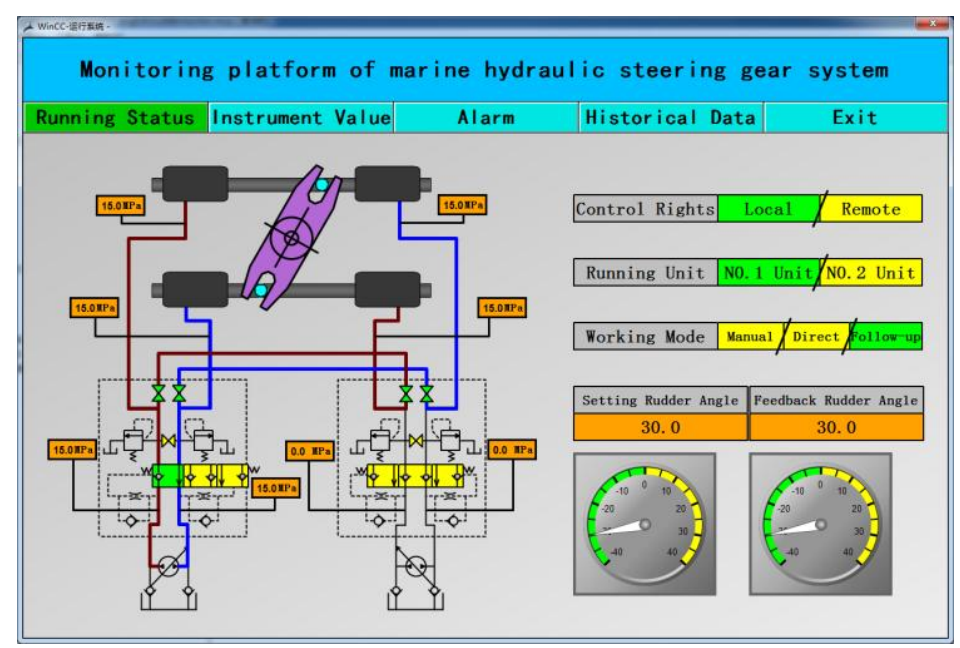

Fig. 1 The "Running Status" interface

(2) The "Instrument Value" interface. For real-time displaying the instrument values when the system is running. As shown in the Fig. 2.

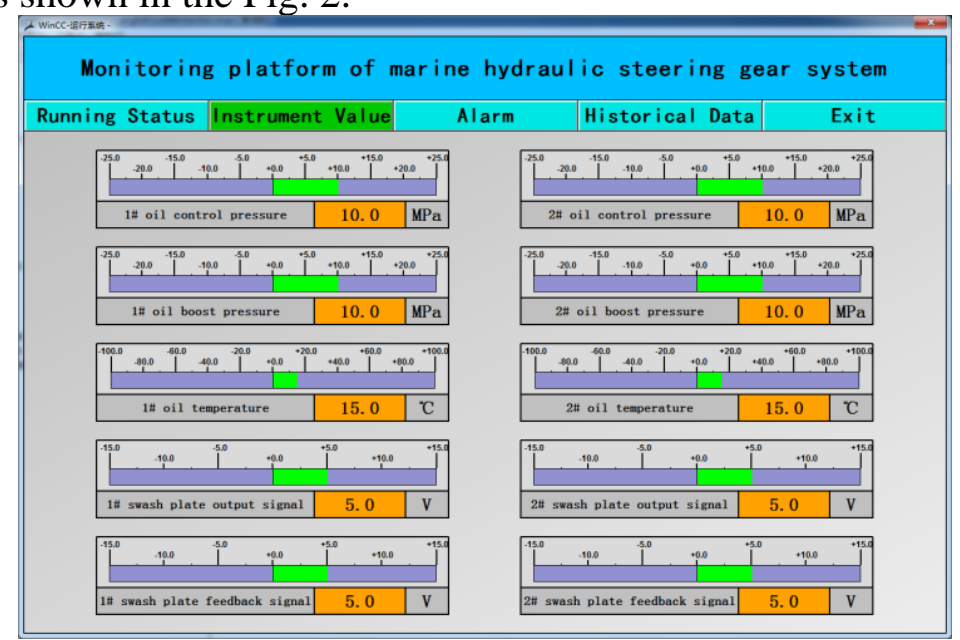

Fig. 2 The "Instrument Value" interface

(3) The "Alarm" interface. Comparing the detected parameters with the set values, if the parameters is abnormal, it will give an alarm. As shown in the Fig. 3.

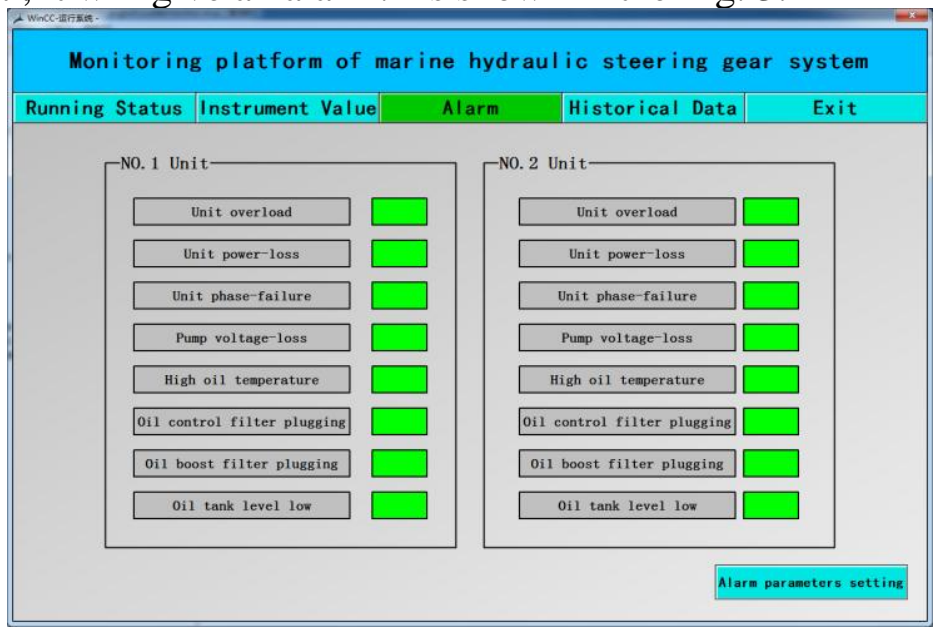

Fig. 3 The "Alarm" interface

(4) The "Historical Data" interface. It is used for displaying and querying for historical data. The historical data can also be printed. As shown in the Fig. 4. 


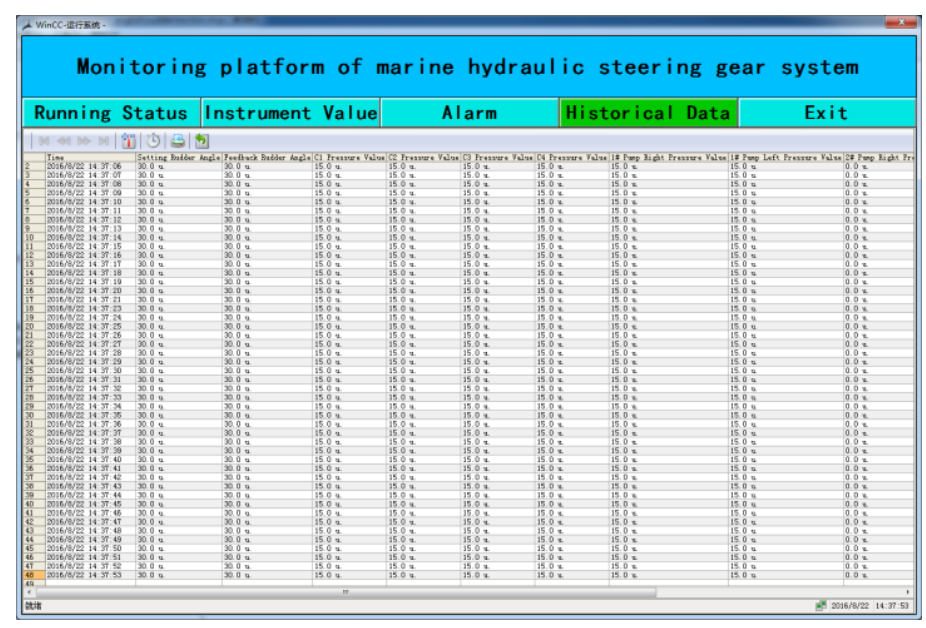

Fig. 4 The "Historical Data" interface

\section{WinCC distributed system}

\subsection{System overview.}

Develop the monitoring system of distributed architecture with configuration software WinCC, this structure will distribute the tasks to the server and the client sides, reduce the communication overhead of the system, and make full use of advantages of the hardware environment on both sides. According to distributing operation and monitoring tasks to multiple servers, balancing the utilized rate of the servers, and allowing a greater improvement in performance of the system. Each of servers can connect up to 32 clients, each of clients can connect up to 12 servers or 12 pairs of redundant servers. In this paper, develop the monitoring system of ship steering gear with configuration software WinCC, which is one of the servers of the ship integrated monitoring system.

\subsection{WinCC server configuration.}

Create a new project of the "multi user project" on the WinCC server computer. Then, configure the necessary data in the project. Second, regist the client in the list of computers, it means that add the client that has permissions to access to the current server to the list of computers, and configure the corresponding parameter properties of the client. Next, in the project, configure the client's landing users and permissions, that is to say, open the user manager and create a client landing user who has the permissions of remoting activation and remote configuration. Finally, the server can generate the server data packet, the packet is a data packet that contains the current configuration data, which is used by all connected clients in the distributed system.

\subsection{WinCC client configuration.}

Create a new project of the "client project" on the WinCC client computer. Then, imported the server data packets so that the data of these WinCC servers can be configured. The finally, configure the pictures of WinCC client.

\section{Conclusion}

In this paper, develop monitoring system of ship steering gear with configuration software WinCC. It is based on network, with the database management for support and the microcomputer for the workstation. The friendly monitoring interface and the stable running system provide the guarantee for the safety of the ship traveling.

\section{References}

[1]. OPC. Programmable controller \& factory automation. (2012) No. 2, p. 70-71.

[2]. Zhang Xueyin: The working principle and management of electro-hydraulic steering gear (Bachelor's degree, Guangdong Ocean University, China 2010). p.5. 
[3]. Nie Anxing. Skillful application of programming to solve the common problems of WinCC configuration. Automation Panorama. (2004) No. 5, p. 43-44.

[4]. Zou Tong. Programming techniques of WinCC configuration software. Control and Instruments in Chemical Industry. (2001) No. 2, p. 21-23.

[5]. Shi Yanxing: Study of the monitoring system for the ship steering-engine (Master's degree, Huazhong University of Science \& Technology, China 2009). p.21.

[6]. Information on: https://support.industry.siemens.com 